\title{
Association of Cognitive Impairment With Migrant Status Among Older Adults in India: A Cross- Sectional Study
}

\section{Shobhit Srivastava}

International Institute for Population Sciences

\section{Ruchi Singh}

Prin. L. N. Welingkar Institute of Management Development and Research

Prem Shankar Mishra ( $\sim$ psmishrabhu@gmail.com )

Institute for Social and Economic Change

\section{Alok Aditya}

Institute for Social and Economic Change

\section{Research Article}

Keywords: Older adults, Cognitive Impairment, Migration, India

Posted Date: August 18th, 2021

DOI: https://doi.org/10.21203/rs.3.rs-745749/v1

License: (a) (i) This work is licensed under a Creative Commons Attribution 4.0 International License. Read Full License 


\section{Abstract}

\section{Introduction}

Among various health implications for older adult, cognitive impairment and related dementias are significant public health concern in many low and middle income countries, including India and lack due attention in policy arena. Socio-economic and health vulnerability are associated with cognitive impairment among older adults. Therefore the present study explores the prevalence and determinants of cognitive impairment among older adults in India with special reference to migrant status of older adults.

\section{Methods}

Data for this study was utilized from recent release of Longitudinal Ageing Study in India (LASI) wave 1 2017-19. The LASI is a nationally representative survey over 72000 older adults age 45 and above across all states and union territories of India. The present study is conducted on the eligible respondent's age 60 years and above. The total sample size for the present study is 31,464 older adults aged 60 years and above (Male-15,068; Female-16,366). Descriptive and logistic regression analysis carried to fulfil the objective of the study.

\section{Results}

Overall, the prevalence of cognitive impairment among male older adults was $6.4 \%$ and female older adults $19.8 \%$. Non-migrant status $(6.8 \%)$ was more likely to face cognitive impairment than migrant status (5.7\%) among older adults. The high prevalence of cognitive impairment were found with increasing socio-economic, demographic and multi-morbidities among older adults. Older adults (male $6.7 \%$ vs. female $20.2 \%$ ) with no social participation were more likely to be face cognitive impairment. The result of logistic regression of our study is supported the bivariate analysis. Older adults with migrant status were more likely to be suffered from the cognitive impairment with unadjusted [UOR; $1.57, \mathrm{Cl}$ : 1.451.70] \& after adjusting with covariates [AOR; 1.14, $\mathrm{Cl}$ : 1.03-1.26] as compared to non-migrant status. Among the individual factors, odds of impairment was very high for the oldest-older adults age group [AOR: 2.95, Cl: 2.59-3.36] as compared to young-older adults and further, female older adults were more likelihood to be cognitive impairment [AOR: 1.99, Cl: 1.77-2.24] than their counterparts. Similar findings were also found with socio-economic and health vulnerability among older adults.

\section{Conclusion}

The study demonstrates that female older adults need more care and support from community and government as they face higher cognitive impairment. Further, the results significantly varied across different socio-economically, demographically, regionally in cognitive impairment and those who were suffering with co-morbidities. Comprehensive primary health care with community health approach may improve the health status of older adults in later period of life. 


\section{Introduction}

Migration is an important phenomenon in demography of any nation. As per National Sample Survey of India (NSSO), roughly 29 percent of population (326 million persons) is internally mobile in India. One third of India's entire population is internal migrant(1). Migration is very complex process and has huge implications, and one of the major implications that often lacks due attention in policy and migration research is mental well-being of an older adults. Migration is an important dimension of global health importance and often have lasting impact on migrants health and well-being of older adults(2). Older migrants comprised an estimated 34.3 million or 12.2 per cent of the international migrant stock at midyear 2020(3). Population aged older adults i.e 60 year and above comprises of 7 percent ( 1.25 billion) of total population in India and it is projected to triple in next four decades (4).

Among various health implications for older adult, cognitive impairment and related dementias are significant public health concern (5). Cognitive impairment is defined as when a person has trouble remembering, learning new things, concentrating, or making decisions that affect their everyday life (Center for Disease Control and prevention's Healthy aging program). Various factors such as socioeconomic status, psychosocial factors, early life condition, genetic factors, behavioral factors, physical and psychological health and environmental factors play important role in ensuring healthy mental being of migrants involved in migration process $(2,6-17)$. Migration process may alter above-mentioned factors and may have negative and positive influence on migrant's cognitive abilities. There is a relationship between migration and cognitive impairment among older adults that need to be understood.

Around 50 million people have dementia worldwide and there are 10 million new cases every year, implying one new case every 3.2 seconds. In India it is estimated that 5.3 million people above the age of 60 have dementia in 2020, which mean one in every 27 person is having dementia, according to the Dementia in India 2020 report. Data shows the burden of congitive impairment is already significant for older adult (18-24). Cognitive impairment is an important age related condition and often brings along numerous another mental ailments such as depression, ADL, IADL, dementia and Alzhiemer's disease and problem indeed is multifactorial (25). WHO mentions cognitive impairment in older adults as one of the critical challenges that need to be addressed at country levels (26). 60 percent of global burden of cognitive impairment are shared by developing countries and more than one-fourth are shared by Chain and India alone (16). India is predicted to have 300 percent increase in prevalence of dementia from 2001-2040 (27). Taking into consideration the burden of cognitive impairment the Government of India has recently committed to spending 2.5 percent of its GDP on health by 2025 . Country has also launched Mental Health Care, Act, 2017 which offers every citizen the right to access mental health care operated or funded by the government $(20,21,28)$. Research shows that prevalence of cognitive impairment is higher in rural and male population $(15,29,30)$. Prevalence of cognitive impairment varies across States in India with 3.5\% in Himachal Pradesh, 5.1 percent in Uttar Pradesh,, 6.5 percent in Kashmir, and 11.5 percent in Kerala (12). 
Though few attempts have been made to understand the association between cognitive impairment and migration at international level $(5,31-34)$, studies on same in Indian context are scarce. Though few studies have been conducted to understand and analyse cognitive impairment in India $(13,15,16,20,21$, $23,27,35-38)$, cognitive health of migrant older adults in India is largely ignored. The impact of disease on older migrants has so far largely been ignored.

With this given background, the study will make an attempt to understand the prevalence and determinants of cognitive impairment in older adult migrants in Indian context. The aim of the study is to determine the factors associated with cognitive impairment among older adults with migrant status in India. The present study hypothesized that there was no association between cognitive impairment and migrant status among older adults in India.

\section{Methods}

\section{Data}

Data for this study was utilized from recent release of Longitudinal Ageing Study in India (LASI) wave 1 (39). LASI is a full-scale national survey of scientific investigation of the health, economic, and social determinants and consequences of population aging in India, conducted in 2017-18 (39). The LASI is a nationally representative survey over 72000 older adults age 45 and above across all states and union territories of India (39). The main objective of the survey is to study the health status and the social and economic well-being of older adults in India. LASI adopted a multistage stratified area probability cluster sampling design to arrive at the eventual units of observation: older adults age 45 and above and their spouses irrespective of age. The survey adopted a three-stage sampling design in rural areas and a fourstage sampling design in urban areas. In each state/UT, the first stage involved the selection of Primary Sampling Units (PSUs), that is, sub-districts (Tehsils/Talukas), and the second stage involved the selection of villages in rural areas and wards in urban areas in the selected PSUs. In rural areas, households were selected from selected villages in the third stage (39). However, sampling in urban areas involved an additional stage. Specifically, in the third stage, one Census Enumeration Block (CEB) was randomly selected in each in urban area (39). In the fourth stage, households were selected from this CEB. The detailed methodology, with the complete information on the survey design and data collection, was published in the survey report. The present study is conducted on the eligible respondent's age 60 years and above. The total sample size for the present study is 31,464 older adults aged 60 years and above (Male-15,068; Female-16,366).

All methods were carried out in accordance with guidelines. We confirm that all experimental protocols were approved by institutional committee and the ethical clearance was provided by Indian Council of Medical Research (ICMR), India.

\section{Variable description}


Cognitive impairment was measured through five broad domains (memory, orientation, arithmetic function, executive function, and object naming). Memory was measured using immediate word recall, delayed word recall; orientation was measured using time and place measure, arithmetic function was measured through backward counting, serial seven, and computation method; executive function was measured through paper folding and pentagon drawing method, and object naming was lastly done to measure the cognitive impairment among older adults (39). A composite score of 0-43 was computed using the domain-wise measure. The lowest 10th percentile is used as a proxy measure of poor cognitive functioning (39).

\section{Explanatory variables}

\section{Main explanatory variable}

The main explanatory variable was migration status among older adult. The variable was assessed using the question "How many years have you been living (continuously) in this area?" If the respondent responded that they were living in this area since birth than he/she was coded as 0 "Non-migrant" and otherwise 1 "Migrant".

\section{Other explanatory variables}

Age was coded as young old (60-69 years), old-old (70-79 years), and oldest-old ( $80+$ years). Sex was coded as male and female. Educational status was coded as no education/primary not completed, primary, secondary and higher. Working status was coded as never worked, currently working, currently not working and retired. Marital status was coded as currently married, widowed, and others. Others included divorced/separated/never married. Social participation was coded as no and yes. Respondents were said to be socially engaged if they participate in the following activities. Eat out of house (Restaurant/Hotel); Go to park/beach for relaxing/entertainment; Play cards or indoor games; Play out door games/sports/exercise/jog/yoga; Visit relatives /friends; Attend cultural performances /shows/Cinema; Attend religious functions / events such as bhajan/satsang/prayer; Attend political/community/organization group meetings; Read books/newspapers/magazines; Watch television/listen radio and Use a computer for e-mail/net surfing etc. If the respondent was involved in any of the above activity, then the respondent was defined to be socially engaged. Physical activity status was coded as frequent (every day), rare (more than once a week, once a week, one to three times in a month), and never. The question through which physical activity was assessed was "How often do you take part in sports or vigorous activities, such as running or jogging, swimming, going to a health center or gym, cycling, or digging with a spade or shovel, heavy lifting, chopping, farm work, fast bicycling, cycling with loads"? (39)

The probable major depression among the older adults with symptoms of dysphoria, calculated using the CIDI-SF (Short Form Composite International Diagnostic Interview) score of 3 or more. This scale estimates a probable psychiatric diagnosis of major depression and has been validated in field settings and widely used in population-based health surveys (39). The lowest 10th percentile is used as a proxy 
measure for major depression among older adults. Self-rated health was coded as good which includes excellent, very good, and good whereas poor includes fair and poor (40). Difficulty in ADL (Activities of Daily Living) was coded as no and yes. Activities of Daily Living (ADL) is a term used to refer to normal daily self-care activities (such as movement in bed, changing position from sitting to standing, feeding, bathing, dressing, grooming, personal hygiene, etc.) The ability or inability to perform ADLs is used to measure a person's functional status, especially in the case of people with disabilities and the ones in their older ages (41). Difficulty in IADL (Instrumental Activities of Daily Living) was coded as no and yes. Activities of daily living that are not necessarily related to the fundamental functioning of a person, but they let an individual live independently in a community. These tasks are necessary for independent functioning in the community. Respondents were asked if they were having any difficulties that were expected to last more than three months, such as preparing a hot meal, shopping for groceries, making a telephone call, taking medications, doing work around the house or garden, managing money (such as paying bills and keeping track of expenses), and getting around or finding an address in unfamiliar places (41).

The monthly per capita consumption expenditure (MPCE) quintile was assessed using household consumption data (39). Sets of 11 and 29 questions on the expenditures on food and non-food items, respectively, were used to canvas the sample households (39). Food expenditure was collected based on a reference period of seven days, and non-food expenditure was collected based on reference periods of 30 days and 365 days (39). Food and non-food expenditures have been standardized to the 30-day reference period (39). The monthly per capita consumption expenditure (MPCE) is computed and used as the summary measure of consumption. The variable was then divided into five quintiles i.e., from poorest to richest (39). Religion was coded as Hindu, Muslim, Christian, and Others. Caste was recoded as Scheduled Tribe, Scheduled Caste, Other Backward Classes (OBC), and others. The Scheduled Castes include the population that is socially segregated and financially/economically weak by their low status as per Hindu caste hierarchy. The Scheduled Tribes (STs) and Scheduled Castes (SCs) are among the most disadvantaged and discriminated socio-economic groups in India (42). The OBC is the group of people who were identified as "educationally, economically and socially backward" (42). The OBC's are considered low in the traditional caste hierarchy but are higher in status than Scheduled Castes. The "other" caste category is identified as having higher social status, mostly belong to upper caste Hindus (43). Place of residence was coded as rural and urban. The regions of India were coded as North, Central, East, Northeast, West, and South.

\section{Statistical approach}

The study used univariate, bivariate and multivariate analysis to fulfil the aim of the objective. Descriptive analysis is used to show the sample profile of the respondents. Further, bivariate analysis was carried out to estimate the prevalence of cognitive impairment among older adults (male-female separately) by selected variables. Further, a proportion test (44) was done to see the difference between male and female older adults in the prevalence of cognitive impairment. The VIF factor was estimated to check multicollinearity and no evidence of multicollinearity was found. Logistic regression (45) was done 
to compare migrants and non-migrants in cognitive impairment in both the ways unadjusted and adjusted odds ratio by selected background characteristics. Adjusted odds ratios are reported in the study with the form of an adjusted odds ratio (AOR) with a 95\% confidence interval (CI).

\section{Result}

Table 1 presents the percentage distribution of the study population aged 60+ (older adults) across different socio-economic, demographic and health backgrounds. The study also looked at between migrants and non-migrants among older adults as it showed $30.7 \%$ of males and $80.3 \%$ of females were migrants. Within the older adults, more than half of the population were in the age group of 60-69 years. Among male older adults, $53 \%$ were having no education or less than primary as compared to $81.4 \%$ of their counterparts (female older adults). Compared to male older adults (16.5\%), female older adults were found $54 \%$ widowed. This shows the high mortality in the late-life years among males compared to females. Compared to male older adults (5.6\%), female older adults were less participated in the social activities. In the current working status, $42 \%$ male older adults and $19 \%$ female older adults were reported as currently working. In the context of health factors, male older adults were $7.2 \%$ found depression as compared to $9.5 \%$ female older adults, however, for self-rated health, male reported $45.3 \%$ as compared to $49 \%$ female older adults. Further, difficulty in ADL found as $21.9 \%$ among male older adults as compared to $26.5 \%$ female older adults. Whereas difficulty in IADL, 39.7\% in male older adults as compared to $56.9 \%$. In MPCE, the female older adults were more deprived and belonged to the poor category than males older adults. The majority of the population was the Hindu community (82\%), followed by $11.7 \%$ Muslim community. In the male older adults, including Scheduled Caste/Scheduled Tribe, 26.5\% were belonged to SC/ST category as compared to $27.5 \%$ SC/ST in the female older adults group. More male older adults (72\%) are living in rural areas as compared to their counterparts (69\%). Regional variation in the distribution of population can be seen as the highest percentage of eastern region (24.6\%) followed by the central region (22.5\%) and south (21.4\%) among male older adults, however, in the female older adults, $23.8 \%$ in the southern region followed by eastern $(22.8 \%)$ and central $(19.6 \%)$.

Table 2 demonstrates the prevalence of cognitive impairment among male and female older adults in India by background characteristics. In cognitive impairment, male older adults with non-migrant status (6.8\%) were highly vulnerable than migrant status (5.7\%). Overall, the prevalence of cognitive impairment among male older adults $6.4 \%$ and female older adults $19.8 \%$. The results indicate that the proportion of females with cognitive impairment were higher than males across all socio-economic and demographic variables. The high prevalence of cognitive impairment found among male respondents aged 80 and above $(15.9 \%)$, having no education $(11.8 \%)$, with no social participation $(6.7 \%)$ with poorest wealth quintile (9.4\%), divorced/separated/never married (10.3\%), having difficulty in ADL (11\%) and IADL (10.7\%), and belong to Scheduled Tribe (14.8\%). Whereas for female older adults with aged 80 and above (41.5\%), having no education (25.1\%), currently not working (26.8\%), widowed (24.6\%), with no social participation (20.2\%), having depression (25.6\%), having difficulty in ADL (29.7\%) and IADL (25.3\%), belonged to poorest MPCE quintile (26.8\%), Scheduled Caste (26.4\%) and Scheduled Tribe (29.3\%) were highly found cognitive impairment. The large difference in prevalence between female and male are 
found in old-old (17.7\%) and oldest-old (25.6\%) age group, currently not working (17.5\%), depressed (18.5\%), having difficulty in ADL (18.7\%), belong to poorest MPCE quintile (17.4\%), and Scheduled caste $(17.8 \%)$.

The result of logistic regression of cognitive impairment on different socio-economic variables, presented in the Table 3 that shows older adults with migrant status were more likely to be suffered from the cognitive impairment with unadjusted [UOR; 1.57, $\mathrm{Cl}$ : 1.45-1.70], after adjusting with covariates [AOR; $1.14, \mathrm{Cl}: 1.03-1.26]$ as compared to non-migrant status. Among the individual factors, odds of impairment was very high for the oldest-older adults age group [AOR: 2.95, Cl: 2.59-3.36] as compared to young-older adults and further, female older adults were more likelihood to be cognitive impairment [AOR: $1.99, \mathrm{Cl}: 1.77-2.24]$ than their counterparts. Lower educational achievement leads to a high chance of cognitive impairment where respondents with no education have a quite high chance of cognitive impairment [AOR: $22.09, \mathrm{Cl}$ : 10.39-46.94] followed by respondents with only primary level education [AOR: 3.61, Cl: 1.65-7.9]. The result shows that older adults currently working [AOR: 0.82, Cl: 0.72-0.94] and retired [AOR: $0.53 \mathrm{Cl}: 0.38-0.75]$ are less likely to suffer from cognitive impairment compared to those older adults who never worked. Compared to currently married, those who were widowed [AOR: 1.37, Cl: 1.25-1.5], and divorced/separated/never married [OR: 1.51, Cl: 1.15-1.97] were more likely to be cognitive impairment. Further those older adults who were having no social participation and never engaged in any physical activities are more likely to be suffered from cognitive impairment.

Among the health factors, older adults with poor self-rated health [AOR: 1.29, Cl: 1.18-1.41] and having difficulty in ADL [AOR: $1.35 \mathrm{Cl}: 1.22-1.50$ ] and IADL [AOR: $1.34 \mathrm{Cl}$ : 1.21-1.47] were more likely to fall under the prevalence of cognitive impairment. Among the household factors, respondents who belonged to the lowest quintiles of MPCE were more likely to be suffered from the cognitive impairment compared to the richest quintile. Further, the older adults who belonged to Scheduled Tribe [AOR: $1.52 \mathrm{Cl}: 1.31-1.76$ ] were more likely while Other Backward Class [AOR: $0.78 \mathrm{Cl}: 0.69-0.88$ ] and Other Caste [AOR: $0.79 \mathrm{Cl}$ : 0.69-0.90] were less likely to suffer from the cognitive impairment compared to Schedule Caste counterparts. Compared to rural resident, the urban residents [OR: $0.49 \mathrm{Cl}: 0.44-0.55]$ were less likely to suffer from cognitive impairment. Further, with reference to north region, central, east and south regions [AOR: $0.86 \mathrm{Cl}: 0.74-0.99]$ were less likely to suffer from cognitive impairment whereas west region [AOR: 1.22 , Cl: 1.04-1.43] was more likely to be suffer from cognitive impairment among older adults.

\section{Discussion}

The present study investigated the prevalence of cognitive impairment by different socio-economic and demographic and health-related factors in both male and female older adults in India. It has also investigated the influence of these factors on the risk of cognitive impairment among older adults. Our major findings are as follow; 1 ) those older adults with migrant status are less likely to be cognitive impairment compare to their counterparts, 2) female older adults were more prone to be affected by cognitive impairment as compared to male older adults, 3) poor socio-economic status of older adults were highly vulnerable in cognitive impairment. 4) Older adults engaged with social activities and 
participation were less prone to cognitive impairment than those who have not participated. 5) Those older adults who were facing co-morbidities such as ADL, IADL, depression and poor health were positively associated with cognitive impairment.

Furthermore, our study supports the existing literature that increasing age leads to mild cognitive impairment $(12,46)$ as we found that moving from young-older adults to old-older adults that increased the prevalence as well as odds of cognitive impairment where oldest-adults are 2.95 times more likely to suffer from cognitive impairment. One plausible explanation is that as the age of the elderly person increases, macular abnormalities, age-related hearing loss increases that in turn increase the risk of cognitive impairment among the older people $(47,48)$. We found in our study that the proportion of females with cognitive impairment is higher than males among all socio-economic and demographic variables. The result shows that 41.5 percent of females belong to the oldest-old age group reported cognitive impairment which supports the prior studies found worse cognitive function in oldest-older aged females compared to males $(49,50)$.

Migration is an underlying factor that leads to cognitive impairment among the migrated people. The global health research found five potential factors that affect the cognitive functioning among the migrated people includes socioeconomic status, psychological factors, behavioural factors, physical and psychological health status, and environmental factors (6). We found that the prevalence differs by migration status in both within and between the male and female populations. The relationship between cognitive impairment and migration depends on many factors where gender has multi-fold influence over this relationship. The reason for the worse cognitive function among the migrated women can be explained through the pattern of migration and its relationship with cognitive functioning. Prior research has found that 70 percent of female migration has rural to rural migration pattern primarily for marriage $(1,51)$ and rural to rural migration has the lowest cognition whereas urban to urban and rural to urban migration has the highest cognition (52).

We found a sharp decline in the prevalence of cognitive impairment when we move from adults with no education to subsequent higher categories of educational achievements. Having no education or not completed primary education increases the odds of cognitive impairment where older adults having no education are 22.9 times more likely to be cognitively impaired than adults who completed higher education. Adults who completed higher education reflect better cognitive function as brain function for such people are stimulated by learning activities (53) and they maintain their brain functioning through the cognitive reserve (54). The prevalence of cognitive impairment is high among the adults suffering from depression, having poor health status, difficulty in ADL and IADL where these factors except depression increase the chance of suffering from cognitive impairment. However, this is not in line with previous studies on the relationship between depression and cognitive functioning which reflect high odds of cognitive impairment for depressed older adults $(55,56)$.

Our result on the cognitive impairment by marital status shows that the prevalence of cognitive impairment was high among single adults and they were more likely to suffer from the impairment than 
adults who were currently married. We also found that the prevalence of cognitive impairment was comparatively high among the female across all marital status. It was lowest among currently married males and highest among divorced/separated/never married males while for females, it was highest among widowed females. This result supports the finding on Chinese (57) and Indian (12) older adults that found marital status affects the cognitive functioning between the male and female differently and differs from the recent finding in West which supports a similar average mental health benefit for both male and female (58).

Further, we found that lower monthly per capita consumption expenditure increases the prevalence of cognitive impairment where the poorest quintile of MPCE has the highest odds of impairment that reduces as we move to the higher quintiles. This supports the literature that low consumption expenditure results in the consumption of low-quality foods with inadequate nutrition that in turn increases the risk of cognitive impairment $(59,60)$. There is association between poverty and cognitive impairment.

Rural males and females were more under the prevalence with a high chance of cognitive impairment compared to their urban counterparts where we found one-fourth of rural females were under the prevalence of cognitive impairment. The result is in line with the previous study found urban residents aged 50 and above had the highest level of cognitive function compared to rural counterparts (61). This result may be explained by the negative impacts of limited access to education, workforce participation, health care, local customs, no social participation, and not engage in any physical activities. We found that these possible reasons increase the chance of impairment which is in line with the growing literature $(55,56,62-65)$.

Our study found a regional disparity in the prevalence of cognitive impairment both within and between males and females. We found that northern, eastern and western regions have high gender disparity in cognitive impairment and belonging to the southern region decreases the odds of impairment. This result is consistent with the previous findings on gender disparity in cognitive health in the northern region (66) and less disadvantage for females in the southern region (50). One plausible explanation is that this may due to the factor education that has a very high influence on the impairment and is in a better position in the southern region (67).

\section{Conclusion}

In conclusion, we have discovered that older adults are more prone to cognitive impairment and also those who are not migrants. Female older adults are more likely to be suffer from cognitive impairment than male older adults. This demonstrates that female older adults need more care and support from community and government. Further, the results significantly varied across different socio-economically, demographically, regionally in cognitive impairment and those who were suffering with multi-morbidities face higher cognitive impairment. Rising levels of multi-morbidity is a serious threat to older adults in terms of cognitive impairment. In India, still huge older adults are lacking essential service coverage. Government must put strategic effort to tackle down the high prevalence of cognitive impairment among 
older adults. Comprehensive primary health care interventions with community approach may improve the health status of older adults in later period of life. Further, social corporate interventions can play an important role in reducing the burden of cognitive impairment among older populations. The study revealed that social participation and activities among older adults have led to reduce cognitive impairment, in this regard, therefore, government initiative on Yoga and Fit India Movement is closely linked to improve the quality of life of older adults.

\section{Declarations}

\section{Ethics approval and consent to participate:}

The study used LASI survey data and LASI data is secondary in nature. The data is freely available on request and survey agencies that conducted the field survey for the data collection have collected a prior consent from the respondent. The ethical clearance was provided by Indian Council of Medical Research (ICMR), India. Therefore ethical approval and consent to participate are not required for this study.

\section{Funding:}

Authors did not received any funding to carry out this research

\section{Consent for publication:}

Not applicable

\section{Availability of data and materials:}

The datasets generated and/or analysed during the current study are available with the International Institute for Population Sciences, Mumbai, India repository and could be accessed from the following link: https://iipsindia.ac.in/sites/default/files/LASI_DataRequestForm_0.pdf. Those who wish to download the data have to follow the above link. This link leads to a data request form designed by International Institute for Population Sciences. After completing the form, it should be mailed to: datacenter@iips.net for further processing. After successfully sending the mail, individual will receive the data in a reasonable time.

\section{Competing Interests}

The authors declare that they have no competing interests.

\section{References}

1. Bhagat, R. B. Urban migration trends, challenges and opportunities in India (World Migr Rep, 2015).

2. Xu, H., Ostbye, T., Vorderstrasse, A. A., Dupre, M. E. \& Wu, B. Place of residence and cognitive function among the adult population in India., 50 (2-3), 119-127 (2018). 
3. International Migrant Stock | Population Division.

4. Bailey, A., Hallad, J. \& James, K. S. 'They had to Go': Indian older adults' experiences of rationalizing and compensating the absence of migrant children. Sustainability, 10 (6), 1946 (2018).

5. Downer, B., Garcia, M. A., Saenz, J., Markides, K. S. \& Wong, R. The role of education in the relationship between age of migration to the United States and risk of cognitive impairment among older Mexican Americans. Res Aging, 40 (5), 411-431 (2018).

6. Xu, H. et al. Migration and cognitive function: a conceptual framework for Global Health Research. Glob Heal Res policy, 3 (1), 1-12 (2018).

7. Schmand, B., Smit, J. H., Geerlings, M. I. \& Lindeboom, J. The effects of intelligence and education on the development of dementia. A test of the brain reserve hypothesis. Psychol Med, 27 (6), 13371344 (1997).

8. Fratiglioni, L., Wang, H-X., Ericsson, K., Maytan, M. \& Winblad, B. Influence of social network on occurrence of dementia: a community-based longitudinal study., 355 (9212), 1315-1319 (2000).

9. Torres, J. M. et al. US migration status of adult children and cognitive decline among older parents who remain in Mexico. Am J Epidemiol, 189 (8), 761-769 (2020).

10. Gubernskaya, Z. Age at migration and self-rated health trajectories after age 50: Understanding the older immigrant health paradox. Journals Gerontol Ser B Psychol Sci Soc Sci, 70 (2), 279-290 (2015).

11. Treas, J. Incorporating immigrants: Integrating theoretical frameworks of adaptation. Journals Gerontol Ser B Psychol Sci Soc Sci, 70 (2), 269-278 (2015).

12. Sengupta, P., Benjamin, A. I., Singh, Y. \& Grover, A. Prevalence and correlates of cognitive impairment in a north Indian elderly population. WHO South-East Asia J public Heal, 3 (2), 135-143 (2014).

13. Selten, J-P., Termorshuizen, F., van Sonsbeek, M., Bogers, J. \& Schmand, B. Migration and dementia: a meta-analysis of epidemiological studies in Europe.Psychol Med. 2020;1-8.

14. Haug, S. Migration networks and migration decision-making. J Ethn Migr Stud, 34 (4), 585-605 (2008).

15. Patel, R. M. \& Singh, U. S. Prevalence study of cognitive impairment and its associated sociodemographic variables using mini-mental status examination among elderly population residing in field practice areas of a medical college. Indian $\mathrm{J}$ community Med Off Publ Indian Assoc Prev Soc Med, 43 (2), 113 (2018).

16. Hill, T. D., Angel, J. L., Balistreri, K. S. \& Herrera, A. P. Immigrant status and cognitive functioning in late-life: An examination of gender variations in the healthy immigrant effect. Soc Sci Med, 75 (12), 2076-2084 (2012).

17. Kopec, A., Ivan Williams, J., To, J. \& Austin, T. C. P. Cross-cultural comparisons of health status in Canada using the Health Utilities Index. Ethn Health, 6 (1), 41-50 (2001).

18. DEMENTIA IN INDIA 20202020. 
19. Raina, S. K. et al. Is dementia differentially distributed? A study on the prevalence of dementia in migrant, urban, rural, and tribal elderly population of Himalayan region in northern India. NAm J Med Sci, 6 (4), 172 (2014).

20. Nulkar, A., Paralikar, V. \& Juvekar, S. Dementia in India-a call for action. J Glob Heal Reports, 3, e2019078-e2019078 (2019).

21. Shaji, K. et al. The Dementia India Report 2010: Prevalence. Impact, Costs Serv Dement. 2010

22. Canevelli, M. et al. Mild Cognitive Impairment in the Migrant Population Living in Europe: An Epidemiological Estimation of the Phenomenon. J Alzheimer's Dis, 73 (2), 715-721 (2020).

23. Khanna, A. B. \& Metgud, C. S. Prevalence of cognitive impairment in elderly population residing in an urban area of Belagavi. J Fam Med Prim Care, 9 (6), 2699 (2020).

24. Samuel, R., McLachlan, C. S., Mahadevan, U. \& Isaac, V. Cognitive impairment and reduced quality of life among old-age groups in Southern Urban India: home-based community residents, free and paid old-age home residents. QJM An Int J Med, 109 (10), 653-659 (2016).

25. Konda, P. R., Sharma, P. K., Gandhi, A. R. \& Ganguly, E. Correlates of Cognitive Impairment among Indian Urban Elders.J Gerontol Geriatr Res. 2018;7(6).

26. World report on Ageing And HeAltH [Internet]. 2015 [cited 2019 Dec 24]. Available from: www.who.int

27. Mohan, D., lype, T., Varghese, S., Usha, A. \& Mohan, M. A cross-sectional study to assess prevalence and factors associated with mild cognitive impairment among older adults in an urban area of Kerala, South India.BMJ Open. 2019;9(3).

28. Dementia in India: An overview | Dementia Care Notes.

29. Poddar, K., Kant, S., Singh, A. \& Singh, T. B. An epidemiological study of dementia among the habitants of eastern Uttar Pradesh, India. Ann Indian Acad Neurol, 14 (3), 164 (2011).

30. Sharma, D., Mazta, S. \& Parashar, A. Prevalence of cognitive impairment and related factors among elderly: A population-based study. J DrNTR Univ Heal Sci, 2 (3), 171 (2013).

31. Garcia, M. A. et al. Age of migration differentials in life expectancy with cognitive impairment: 20year findings from the Hispanic-EPESE. Gerontologist, 58 (5), 894-903 (2018).

32. Jauny, R., Neville, S., Montayre, J. \& Clair, W-S. What is the lived experience of older migrants with mild cognitive impairment. 2018

33. Weden, M. M. et al. The Hispanic paradox: Race/ethnicity and nativity, immigrant enclave residence and cognitive impairment among older US adults. J Am Geriatr Soc, 65 (5), 1085-1091 (2017).

34. Burns, A. \& Zaudig, M. Mild cognitive impairment in older people., 360 (9349), 1963-1965 (2002).

35. Sagbakken, M., Spilker, R. S. \& Ingebretsen, R. Dementia and migration: Family care patterns merging with public care services. Qual Health Res, 28 (1), 16-29 (2018).

36. Raina, S., Razdan, S., Pandita, K. K. \& Raina, S. Prevalence of dementia among Kashmiri migrants. Ann Indian Acad Neurol, 11 (2), 106 (2008).

37. Kang, G., Hajduk, A., Marottoli, R. \& Nunez-Smith, M. Older immigrants perceived health after migration to the United States: Influence of age and level of acculturation.J Am Geriatr Soc. 2021 
38. Gao, Y. et al. Psychological and cognitive impairment of long-term migrators to high altitudes and the relationship to physiological and biochemical changes. Eur J Neurol, 22 (10), 1363-1369 (2015).

39. International Institute for Population Sciences (IIPS), NPHCE. MoHFW HTHCS of PH (HSPH) and the U of SC (USC). Longitudinal Ageing Study in India (LASI) Wave 1, 2017-18, India Report (Mumbai., 2020).

40. Heistaro, S., Jousilahti, P., Lahelma, E., Vartiainen, E. \& Puska, P. Self rated health and mortality: A long term prospective study in eastern Finland.J Epidemiol Community Health. 2001

41. Muhammad, T. \& Srivastava, S. Why Rotational Living Is Bad for Older Adults ? Evidence from a Cross- Sectional Study in India Why Rotational Living Is Bad for Older Adults ? Evidence from a Cross-Sectional Study in India. 2020;(October).

42. Subramanian, S. V. et al. The mortality divide in India: The differential contributions of gender, caste, and standard of living across the life course.Am J Public Health. 2006

43. Deshpande, M. S. History of the Indian Caste System and Its impact on India Today. Senior project. 2010.

44. Fan, C., Wang, L. \& Wei, L. Comparing Two Tests for Two Rates.Am Stat. 2017

45. Osborne, J. \& King, J. E.. Binary Logistic Regression. In: Best Practices in Quantitative Methods.SAGE Publications, Inc.; 2011.p. 358-84.

46. Celsis, P. Age-related cognitive decline, mild cognitive impairment or preclinical Alzheimer's disease? Ann Med, 32 (1), 6-14 (2000).

47. Clemons, T. E., Rankin, M. W. \& McBee, W. L. Cognitive impairment in the age-related eye disease study: AREDS report no. 16. Arch Ophthalmol, 124 (4), 537-543 (2006).

48. Panza, F. et al. Sensorial frailty: age-related hearing loss and the risk of cognitive impairment and dementia in later life. Ther Adv Chronic Dis, 10, $2040622318811000-2040622318811000$ (2019).

49. Ferri, C. P. et al. Global prevalence of dementia: a Delphi consensus study., 366 (9503), 2112-2117 (2005).

50. Mathuranath, P. S. et al. Dementia in Kerala, South India: prevalence and influence of age, education and gender. Int J Geriatr Psychiatry A J psychiatry late life allied Sci, 25 (3), 290-297 (2010).

51. Mohapatra, S. Almost a third of Indians, or over 300 million people, are migrants.

52. Xu, H., Dupre, M. E., Ostbye, T., Vorderstrasse, A. \& Wu, B. Association between Migration and Cognitive Function among Older Adults in China. Innov Aging, 1 (Suppl 1), 1278 (2017).

53. Fratiglioni, L., Paillard-Borg, S. \& Winblad, B. An active and socially integrated lifestyle in late life might protect against dementia. Lancet Neurol, 3 (6), 343-353 (2004).

54. Valenzuela, M. J. \& Sachdev, P. Brain reserve and dementia: a systematic review. Psychol Med, 36 (4), 441-454 (2006).

55. Muhammad, T. \& Meher, T. Association of late-life depression with cognitive impairment: evidence from a cross-sectional study among older adults in India. BMC Geriatr, 21 (1), 1-13 (2021). 
56. Aajami, Z. Relationship between depression and cognitive impairment among elderly: A crosssectional study. J Caring Sci, 9 (3), 148 (2020).

57. Feng, L. et al. Marital Status and Cognitive Impairment among Community-Dwelling Chinese Older Adults: The Role of Gender and Social Engagement (Dement Geriatr Cogn Dis Extra, 2014).

58. Williams, K., Frech, A. \& Carlson, D. L. Marital status and mental health.A Handb study Ment Heal Soc Context Theor Syst. 2010;306-20.

59. González-Gross, M., Marcos, A. \& Pietrzik, K. Nutrition and cognitive impairment in the elderly. Br J Nutr, 86 (3), 313-321 (2001).

60. Scarmeas, N., Anastasiou, C. A. \& Yannakoulia, M. Nutrition and prevention of cognitive impairment. Lancet Neurol, 17 (11), 1006-1015 (2018).

61. Xu, H., Dupre, M. E., Gu, D. \& Wu, B. The impact of residential status on cognitive decline among older adults in China: results from a longitudinal study. BMC Geriatr, 17 (1), 1-11 (2017).

62. Lee, J., Shih, R., Feeney, K. \& Langa, K. M. Gender disparity in late-life cognitive functioning in India: findings from the longitudinal aging study in India. Journals Gerontol Ser B Psychol Sci Soc Sci, 69 (4), 603-611 (2014).

63. Zhang, Z., Gu, D. \& Hayward, M. D. Early life influences on cognitive impairment among oldest old Chinese. Journals Gerontol - Ser B Psychol Sci Soc Sci, 63 (1), 25-33 (2008).

64. Laurin, D., Verreault, R., Lindsay, J., MacPherson, K. \& Rockwood, K. Physical activity and risk of cognitive impairment and dementia in elderly persons. Arch Neurol, 58 (3), 498-504 (2001).

65. Yen, Y., Yang, M., Shih, C. \& Lung, F. Cognitive impairment and associated risk factors among aged community members. Int J Geriatr Psychiatry, 19 (6), 564-569 (2004).

66. Ganguli, M. et al. Cognitive test performance in a community-based nondemented elderly sample in rural India: the Indo-US Cross-National Dementia Epidemiology Study. Int Psychogeriatrics, 8 (4), 507-524 (1996).

67. Husain, Z. Gender disparities in completing school education in India: explaining geographical variations. J Popul Res, 28 (4), 325 (2011).

\section{Tables}

Due to technical limitations, table 1-3 is only available as a download in the Supplemental Files section.

\section{Supplementary Files}

This is a list of supplementary files associated with this preprint. Click to download.

- Tables.pdf 